\title{
SAR and thermal response effects of a two- arm Archimedean spiral coil in a magnetic induction sensor on a human head
}

\author{
Ziyi Zhang ${ }^{\mathrm{a}, \mathrm{b}, *}$, Peiguo Liu ${ }^{\mathrm{b}}$, Dongming Zhou ${ }^{\mathrm{b}}$, Liang Zhang ${ }^{\mathrm{a}, \mathrm{b}}$ and Liang Ding ${ }^{\mathrm{b}}$ \\ ${ }^{a}$ College of Science, National University of Defense Technology, Changsha, Hunan 410073, China \\ ${ }^{\mathrm{b}}$ State Key Laboratory of Complex Electromagnetic Environmental Effects on Electronics \& \\ Information System, National University of Defense Technology, Changsha, Hunan 410073, China
}

\begin{abstract}
This study investigates the radiation safety of a newly designed magnetic induction sensor. This novel magnetic induction sensor uses a two-arm Archimedean spiral coil (TAASC) as the exciter. A human head model with a real anatomical structure was used to calculate the specific absorption rate (SAR) and temperature change. Computer Simulation Technology (CST) was used to determine the values of the peak 10-g SAR under different operating parameters (current, frequency, horizontal distance between the excitation coil and the receiver coil, vertical distance between the top of the head model and the $X O Y$ plane, position of excitation coil, and volume of hemorrhage). Then, the highest response for the SAR and temperature rise was determined. The results showed that this new magnetic induction sensor is safe in the initial state; for safety reasons, the TAASC current should not exceed $4 \mathrm{~A}$. The scalp tissue absorbed most of the electromagnetic energy. The TAASC's SAR/thermal performance was close to that of the circular coil.
\end{abstract}

Keywords: Specific absorption rate (SAR), temperature rise, magnetic induction, two-arm Archimedean spiral coil (TAASC), human head model

\section{Introduction}

Recently, magnetic induction measuring has attracted growing interest due to its intracerebral hemorrhage (ICH) evaluation [1, 2]. Magnetic induction measurements can be obtained by placing the coils around a person's head. The current frequency in the excitation coil usually ranges from tens of $\mathrm{kHz}$ to several $\mathrm{MHz}$ [3], and is even higher in some studies [4]. Radiation safety assessment should be performed when a human head is exposed to the RF electromagnetic fields. The person's head can absorb the transmitting power from the excitation coil, which will in turn cause a temperature increase in the person's head. The specific absorption rate (SAR) is a widely used indicator that can reflect the quantity of the absorbed electromagnetic energy within the unit mass of human tissue; the temperature rise positively correlates with the SAR. According to the IEEE standard, the safety threshold for the

\footnotetext{
*Address for correspondence: Ziyi Zhang, Room 605, Electromagnetic Environment Laboratory Building, College of Electronic Science and Engineering, National University of Defense Technology, 109 Deya Road, Kaifu District, Changsha, Hunan 410073, China. Tel.: +86 073184576219 15; Fax: +86 0731 84576219; E-mail: ziyizhang@nudt.edu.cn.
}

0959-2989/15/\$35.00 @ 2015 - IOS Press and the authors. 
SAR is $2.0 \mathrm{~W} / \mathrm{kg}$ over 10 gram of tissue [5]. The electromagnetic field numerical approaches such as the method of moments (MoM) [6] and the finite-difference time-domain (FDTD) method [7], are frequently-used to estimate the SAR. The SAR quantification can also be simulated by the commercial electromagnetic simulation software which employs these techniques [8]. A significant amount of research has been conducted on SAR and the thermal effect of mobile phone, antenna, and MRI on the human brain [9-11]. However, due to the facts that magnetic induction measurement is an emerging technique and its excitation frequency is relatively low, the SAR and thermal response are ignored in many of these studies. To the authors' knowledge, the only work of this field has been reported by Zolgharni, et al [12]. This study addresses the radiation safety of the newly designed magnetic induction sensor. This novel magnetic induction sensor uses a two-arm Archimedean spiral coil (TAASC) as the exciter. The SAR and temperature rise are calculated by the commercial software Computer Simulation Technology (CST). A human head model with real anatomical structure was placed in the coil system to conduct the simulation experiments. The results were compared with those of Zolgharni, et al.

\section{Model and simulation}

\subsection{Novel magnetic induction sensor}

In magnetic induction measurements, the conventional exciter is the circular coil that can emit primary electromagnetic fields in space. The major drawback of the circular excitation coil is that primary fields are considerably more intense than those of secondary fields. To overcome this difficulty, the authors of this study propose a novel two-arm Archimedean spiral coil (TAASC) exciter. In previous publications, the authors verified that the TAASC can counteract its primary magnetic fields at the receiver coil and consequently improve the measurement sensitivity compared with the conventional circular excitation coil [13]. As shown in Figure 1(a), the TAASC is constructed by two opposite Archimedean spirals connected in the center. Both spirals have 6 turns with the maximum outer radium of $50 \mathrm{~mm}$. The receiver coil is a single-turn circular coil with $50 \mathrm{~mm}$ radium. The TAASC and the receiver coil are coaxially located in the $Y Z$ plane, and their axis is coincident with the $x$-axis. The two coils are the same distance from the YOZ plane, and their distance is denoted as the horizontal distance. The wire diameter of the two coils is $1 \mathrm{~mm}$. In the rectangular coordinate system, the parametric equations of the two spirals of TAASC (unit: $\mathrm{mm}$ ) are:

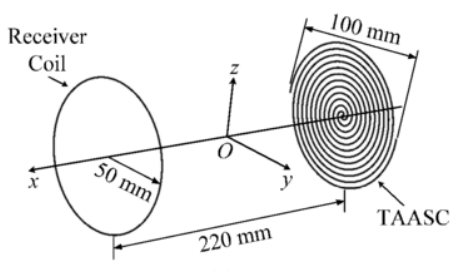

(a)

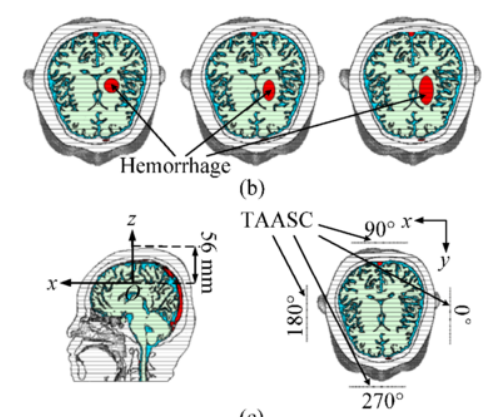

(c)

Fig. 1. Illustration of the model. (a) Configuration of the novel magnetic induction sensor. (b) Three types of ICH: the mild case (left), the moderate case (middle), and the severe case (right). (c) The position of the head model (left), and four sites where the excitation coil can be located (right). 


$$
\left\{\begin{array} { l } 
{ y _ { \text { spiral } 1 } = \frac { 5 0 } { 1 2 \pi } \varphi \operatorname { c o s } \varphi } \\
{ z _ { \text { spiral } 1 } = \frac { 5 0 } { 1 2 \pi } \varphi \operatorname { s i n } \varphi }
\end{array} \quad \left\{\begin{array}{l}
y_{\text {spiral } 2}=\frac{50}{12 \pi} \varphi \cos (\varphi-\pi) \\
z_{\text {spiral } 2}=\frac{50}{12 \pi} \varphi \sin (\varphi-\pi)
\end{array}\right.\right.
$$

where the parameter $\varphi$ is in the range of 0 to $12 \pi$.

\subsection{Human head model}

The human head model, provided by Third Military Medical University, is developed from the second Chinese visible human $(\mathrm{CVH})$ data. It is a three-dimensional model with a real anatomical structure, and can be imported to CST to calculate the SAR and temperature rise. The human head model contains seven tissues, including scalp, skull, cerebrospinal fluid (CSF), brain, optic nerve, eye, and blood. The hemorrhage can be added to the head model. The site where the hemorrhage is located usually appears in the left or right hemisphere of the brain and above the eye level. This paper addresses three types of ICH: a mild case with a volume of $4.2 \mathrm{ml}$, a moderate case with a volume of $18.8 \mathrm{ml}$, and a severe case with a volume of $31.4 \mathrm{ml}$. As shown in Figure 1(b), all three cases appear in the left hemisphere of the brain.

\subsection{SAR and temperature rise calculation}

The proper passive electrical and thermal properties were assigned to tissues in the head model for the SAR and temperature rise calculation [14-18]. Muscle and white matter respectively compose most of the scalp and brain, so the physical properties of the scalp and brain are nearly identical to those of muscle and white matter, respectively. The physical properties of the skull and eyeball were valued using the weighted average, and the physical properties of the hemorrhage were estimated by assuming that the lesion contained $75 \%$ blood. The tissues were assumed to be uniform, linear, and isotropic mediums. The human head model was placed in the sensor in Figure 1(a), with its vertical axis at the $z$-axis and faced toward the $y$-axis. The distance between the top of the head model and the $X O Y$ plane was denoted as the vertical distance, as shown in Figure 1(c).

This new magnetic induction sensor's operating parameters were chosen as the initial state. It was a system with 1 A current, $10 \mathrm{MHz}$ frequency, $220 \mathrm{~mm}$ distance between the excitation coil and the receiver coil, $56 \mathrm{~mm}$ distance between the top of the head model and the XOY plane (see Figure 1(c)), $0^{\circ}$ position for the excitation coil (see Figure 1(c)), and a mild hemorrhage. Simulation experiment proceeded with changing one factor at a time. The selected samples for the current were $1 \mathrm{~A}, 2 \mathrm{~A}, 3 \mathrm{~A}$, $4 \mathrm{~A}, 5 \mathrm{~A}$, and $10 \mathrm{~A}$; frequencies were $10 \mathrm{MHz}, 15 \mathrm{MHz}$, and $20 \mathrm{MHz}$; distances between the excitation coil and the receiver coil were $220 \mathrm{~mm}, 250 \mathrm{~mm}$, and $280 \mathrm{~mm}$; distances between the top of the head model and the $X O Y$ plane were $51 \mathrm{~mm}, 53.5 \mathrm{~mm}, 56 \mathrm{~mm}, 58.5 \mathrm{~mm}$ and $61 \mathrm{~mm}$; excitation coil positions were $0^{\circ}, 90^{\circ}, 180^{\circ}$, and $270^{\circ}$; hemorrhage volumes were $4.2 \mathrm{ml}, 18.8 \mathrm{ml}$, and $31.4 \mathrm{ml}$. The highest response for the SAR and temperature rise was determined. The SAR and temperature rise were calculated by CST MICROWAVE and MPHYSICS STUDIO, respectively. The background and body core temperature were set as $24^{\circ} \mathrm{C}$ and $37^{\circ} \mathrm{C}$, respectively.

\section{Results and discussions}




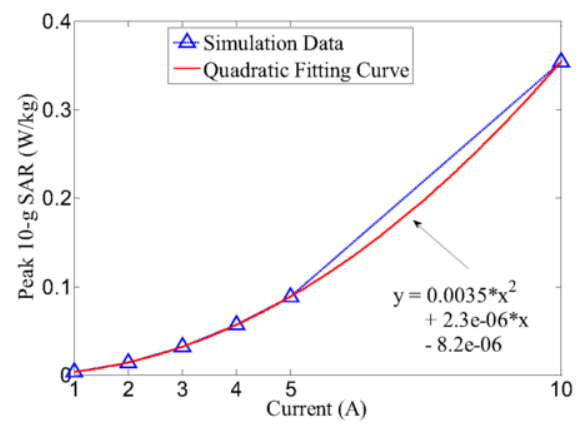

(a)

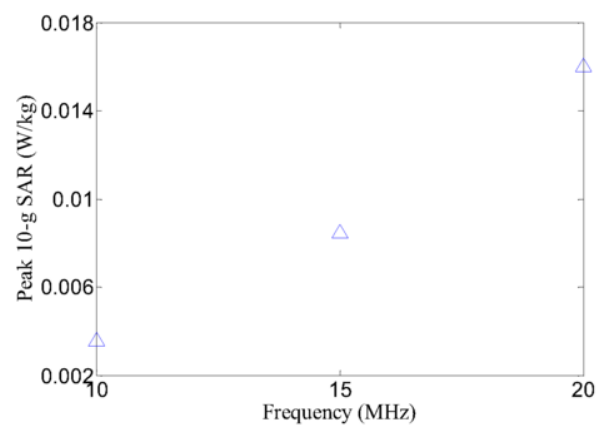

(b)

Fig. 2. Peak 10-g SAR versus the current (a) and frequency (b) of the exciter.

\subsection{Peak 10-g SAR versus current and frequency}

Figure 2 shows the peak 10-g SAR versus the current and frequency of the exciter. The SAR tended to grow with the current and frequency; hence, increasing the current and frequency will enlarge the SAR. Figure 2(a) depicts the quadratic fitting curve based on the simulation data. The fitting equation is $y=0.0035 x^{2}+2.3 \times 10^{-6} x-8.2 \times 10^{-6}$, and the norm of residuals is $1.5191 \times 10^{-5}$. The fitting curve reflects the variation regularity of the simulation data, implying that the SAR is almost proportional to the square of the current. In fact, this quadratic relation is reasonable as the SAR is defined to be proportional to the square of the electric field intensity, and the excitation current is proportional to the space electric field intensity theoretically. The peak 10-g SAR for the initial state was $3.5399 \times 10^{-3}$ $\mathrm{W} / \mathrm{kg}$, which is almost three orders of magnitude lower than the safety threshold of the IEEE standard and will cause an insignificant temperature increase. Thus, in the initial state, the novel magnetic induction sensor was harmless. This value was almost equal to the SAR (around $3 \times 10^{-3} \mathrm{~W} / \mathrm{kg}$ ) computed by Zolgharni, et al. based on their magnetic induction sensor. As Zolgharni, et al. employ the circular excitation coil and their operating parameters were approximate with ours, it can be concluded that the TAASC's SAR/thermal performance is close to that of the circular coil.

\subsection{Peak 10-g SAR versus horizontal and vertical distance}

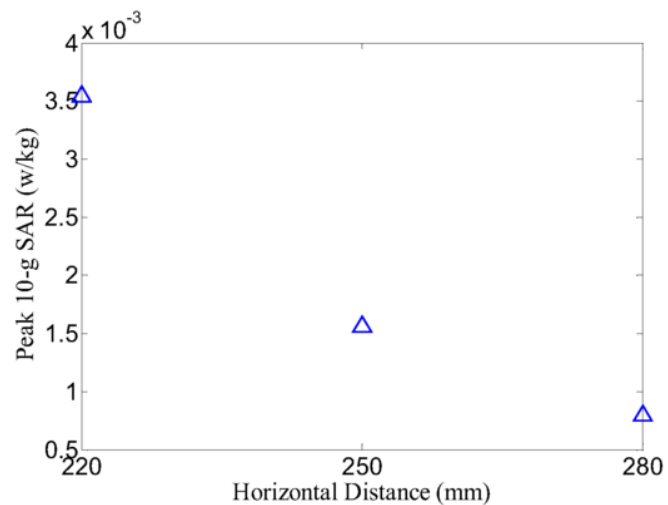

(a)

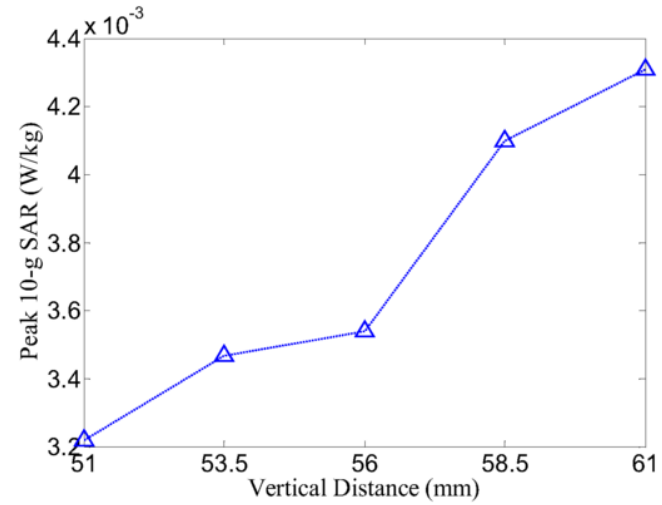

(b)

Fig. 3. Peak 10-g SAR versus the horizontal distance between the excitation coil and the receiver coil (a) and the vertical distance between the top of the head model and the XOY plane (b). 


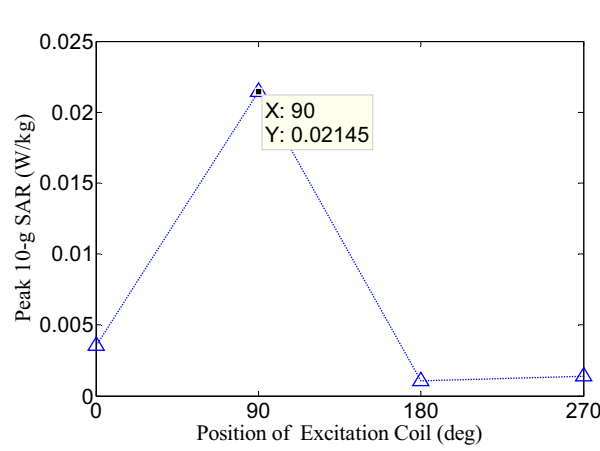

(a)

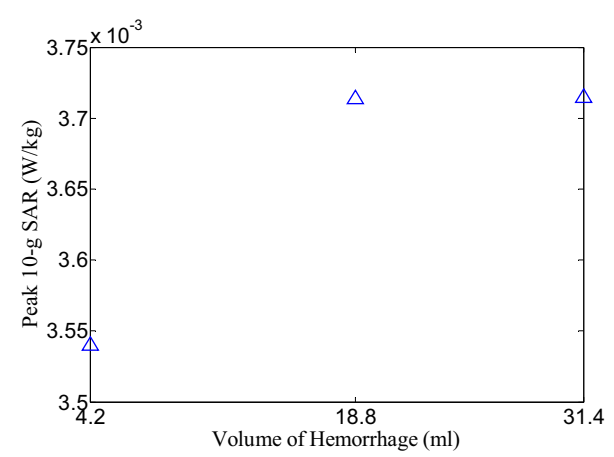

(b)

Fig. 4. Peak 10-g SAR versus the excitation coil position (a) and hemorrhage volume (b).

Figures 3(a) and 3(b) show the peak 10-g SAR versus the horizontal distance between the excitation coil and the receiver coil, and the vertical distance between the top of the head model and the XOY plane, respectively. From the figure, it is evident that the SAR decreases as the horizontal distance increases, while it increases as the vertical distance increases. Considering these two parameters of the horizontal and vertical distance in the novel magnetic induction sensor, the highest response for the SAR is that the horizontal distance between the excitation coil and the receiver coil is $220 \mathrm{~mm}$ and the vertical distance between the top of the head model and the XOY plane is $61 \mathrm{~mm}$.

\subsection{Peak 10-g SAR versus exciter position and blood volume}

Figure 4 displays the peak 10-g SAR versus the excitation coil position and the hemorrhage volume. As demonstrated in Figure 4(a), there existed no explicit relationship between the SAR and the exciter position. The maximum peak $10-\mathrm{g}$ SAR was $0.02145 \mathrm{~W} / \mathrm{kg}$, which appeared at the $90^{\circ}$ position; it was almost ten times those of the other three simulation datasets. The variation of the peak SAR values was mainly caused by the difference of electrical conductivity and material density distribution in the head model; it also related to the geometry of the head model. From Figure 4(b), as the blood volume increased, the SAR increased obviously at first, and then became almost unchanged. For the moderate ICH $(18.8 \mathrm{ml})$, the peak 10 -g SAR was $3.7126 \times 10^{-3} \mathrm{~W} / \mathrm{kg}$. For the severe ICH $(31.4 \mathrm{ml})$, the peak 10 -g SAR was $3.7142 \times 10^{-3} \mathrm{~W} / \mathrm{kg}$. When the hemorrhage volume was larger, the equivalent electrical conductivity of the lesion also increased. Theoretically, as the SAR was proportional to the electrical conductivity, the increase in blood volume enlarged the SAR. However, due to the discretization errors of the software, this growth feature was not presented well. The errors could be reduced by refining the mesh.

\subsection{Highest response for SAR and temperature rise}

The above data was used to determine the highest response for the SAR and the temperature rise for the new magnetic induction sensor: 10 A current, $20 \mathrm{MHz}$ frequency, $220 \mathrm{~mm}$ horizontal distance between the excitation coil and the receiver coil, $61 \mathrm{~mm}$ vertical distance between the top of the head model and the $X O Y$ plane, $90^{\circ}$ position of excitation coil, and severe hemorrhage. In the real magnetic induction sensor, among all the factors discussed, the excitation current was the most flexible parameter; it could change the SAR and increase the temperature easily and greatly. Table 1 gives the values of peak 10-g SAR and peak temperature rise $(\Delta T)$ of the highest response with respect to the 
Table 1

Values of peak 10-g SAR and peak temperature rise $(\Delta T)$ of the highest response with respect to the excitation currents

\begin{tabular}{lllllll}
\hline \multirow{2}{*}{ Highest Response Variable } & \multicolumn{7}{c}{ Excitation Current (A) } \\
\cline { 2 - 7 } & 1 & 2 & 3 & 4 & 5 & 6 \\
\hline Peak 10-g SAR $(\mathrm{W} / \mathrm{kg})$ & 0.11 & 0.42 & 0.95 & 1.69 & 2.64 & 10.71 \\
Peak $\Delta T\left(\times 10^{-3}{ }^{\circ} \mathrm{C}\right)$ & 1.137 & 1.140 & 1.152 & 1.163 & 1.182 & 1.320 \\
\hline
\end{tabular}

different excitation currents. The initial temperature for the head model can be obtained when there was no current in the excitation coil. From the table, it can be seen that the SAR of the highest response exceeded $2 \mathrm{~W} / \mathrm{kg}$ when the excitation current exceeded $5 \mathrm{~A}$. Similar to the SAR, the peak $\Delta T$ of the highest response presented the approximate quadratic relation with the excitation current as well. The temperature variation was quite small, but for safety reasons the excitation current should not exceed $4 \mathrm{~A}$.

Figure 5 shows the distributions of 10-g SAR and the temperature of the highest response at $5 \mathrm{~A}$ current. As shown in Figures 5(a) and 5(b), the maximum SAR value was 2.64, which was slightly more than the safety threshold, and was located in the scalp tissue. The SAR values were much larger at the surface of the head model in the vicinity of the excitation coil. This indicates that most of the electromagnetic energy was absorbed by the scalp tissue, and only a small fraction of the energy

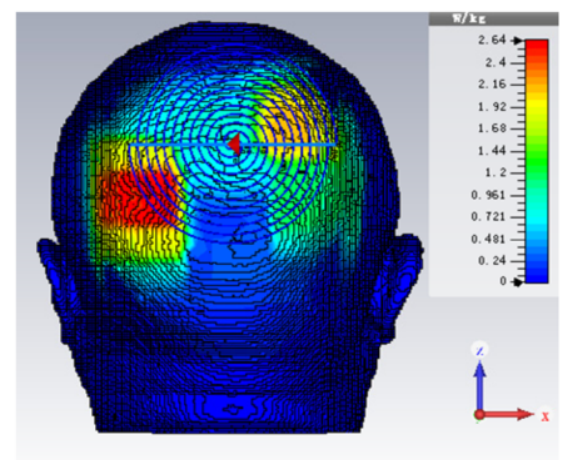

(a)

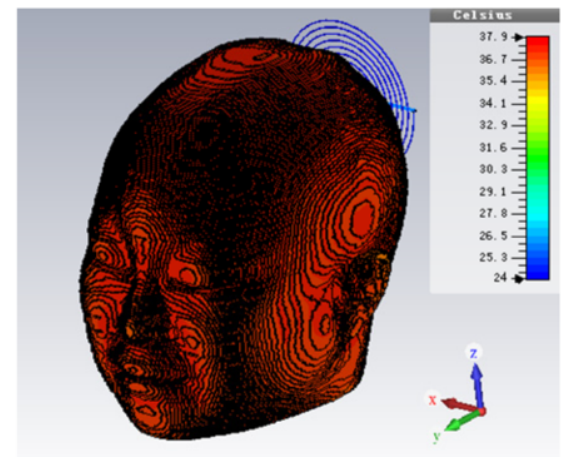

(c)

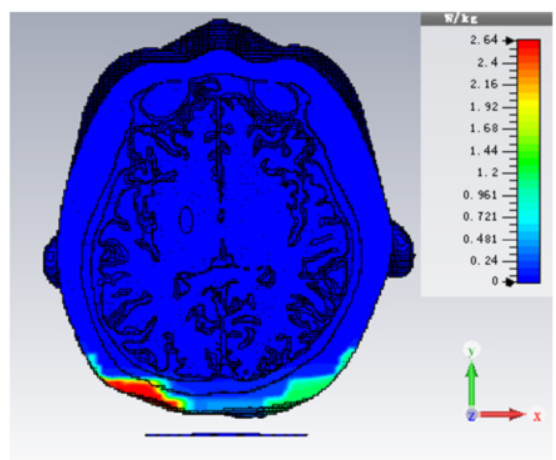

(b)

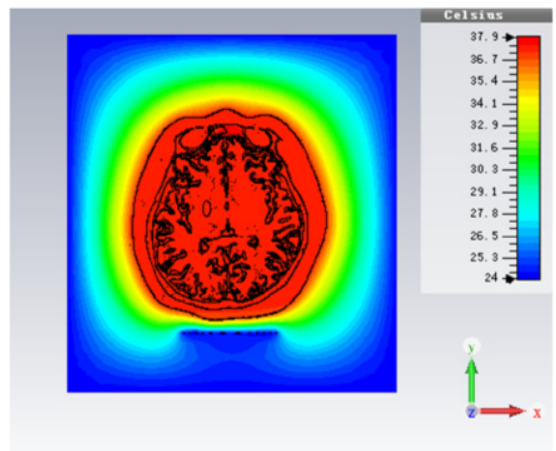

(d)

Fig. 5. Distribution maps of 10-g SAR and temperature of the highest response with 5 A current. (a) Surface view of the SAR distribution. (b) Cross-sectional view of the SAR distribution. (c) Surface view of the temperature distribution. (d) Crosssectional view of the temperature distribution. 
penetrated the scalp to be absorbed by other tissues in the head. This characteristic of electromagnetic energy absorption coincides with the results of Zolgharni, et al. In Figures 5(c) and 5(d), the distribution of the temperature in the head model was relatively uniform. The maximum value of the temperature was about $37.91586^{\circ} \mathrm{C}$, and was also located in the scalp tissue.

\section{Conclusion}

In this paper, simulation experiments were used to study the SAR and thermal response for the human head due to the TAASC in the magnetic induction sensor. Six factors that could influence the SAR and temperature rise were considered. The results showed that our magnetic induction sensor was safe in the initial state, and for safety reasons the current of the TAASC should not exceed 4 A. The scalp tissue absorbed most of the electromagnetic energy. The experiment also demonstrated that the SAR/thermal performance of the TAASC was close to that of the circular coil.

\section{Acknowledgment}

This work was supported by the Innovation Fund for Graduates from the Hunan Province Education Office under Grant CX2013B011, and the Innovation Fund for Doctoral Candidates from the National University of Defense Technology under Grant B130205. The authors would like to thank the Associate Professor X. Ning from Third Military Medical University and the Engineer H. Li from No. 161 Hospital of PLA for helpful discussions, and are also grateful to Third Military Medical University for providing the Chinese head model.

\section{References}

[1] H. Wei and M. Soleimani, Electromagnetic tomography for medical and industrial applications: challenges and opportunities, Proceedings of the IEEE 101 (2013), 27-46.

[2] G. Jin, J. Sun, M. Qin, Q. Tang, L. Xu, X. Ning, J. Xu, X. Pu and M. Chen, A new method for detecting cerebral hemorrhage in rabbits by magnetic inductive phase shift, Biosensors and Bioelectronics 52 (2014), 374-378.

[3] H. Wei and M. Soleimani, Hardware and software design for a National Instrument-based magnetic induction tomography system for prospective biomedical applications, Physiological Measurement 33 (2012), 863-879.

[4] R. Rojas, B. Rubinsky and C. González, The effect of brain hematoma location on volumetric inductive phase shift spectroscopy of the brain with circular and magnetron sensor coils: A numerical simulation study, Physiological Measurement 29 (2008), S255-S266.

[5] IEEE Recommended Practice for Measurements and Computations of Radio Frequency Electromagnetic Fields With Respect to Human Exposure to Such Fields, $100 \mathrm{kHz}-300 \mathrm{GHz}$, The Institute of Electrical and Electronics Engineers, Inc., New York, 2002.

[6] J. Chakarothai, J. Wang, O. Fujiwara, K. Wake and S. Watanabe, Dosimetry of a reverberation chamber for whole-body exposure of small animals, IEEE Transactions on Microwave Theory and Techniques 61 (2013), 3435-3445.

[7] V. Singh, A. Qusba, A. Roy, R. Castro, K. McClure, R. Dai, R. Greenberg, J. Weiland, M. Humayun and G. Lazzi, Specific absorption rate and current densities in the human eye and head induced by the telemetry link of an epiretinal prosthesis, IEEE Transactions on Antennas and Propagation 57 (2009), 3110-3118.

[8] E. Chow, M. Morris and P. Irazoqui, Implantable RF medical devices, IEEE Microwave Magazine 14 (2013), 64-73.

[9] S. Leung, Y. Diao, K. Chan, Y. Siu and Y. Wu, Specific absorption rate evaluation for passengers using wireless communication devices inside vehicles with different handedness, passenger counts, and seating locations, IEEE Transactions on Biomedical Engineering 59 (2012), 2905-2912.

[10] C. Lee and C. Jung, Radiation-pattern-reconfigurable antenna using monopole-loop for fitbit flex wristband, IEEE 
Antennas and Wireless Propagation Letters 14 (2015), 269-272.

[11] B. Park, S. Rajan, J. Guag and L. Angelone, A novel method to decrease electric field and SAR using an external high dielectric sleeve at $3 \mathrm{~T}$ head MRI: numerical and experimental results, IEEE Transactions on Biomedical Engineering 62 (2015), 1063-1069.

[12] M. Zolgharni, P. Ledger and H. Griffiths, Forward modelling of magnetic induction tomography: a sensitivity study for detecting haemorrhagic cerebral stroke, Medical \& Biological Engineering \& Computing 47 (2009), 1301-1313.

[13] Z. Zhang, P. Liu, D. Zhou, L. Zhang and H. Lei, Intracerebral hemorrhage (ICH) evaluation with a novel magnetic induction sensor: a preliminary study using the Chinese head model, Bio-Medical Materials and Engineering 24 (2014), 3579-3587.

[14] S. DeMarco, G. Lazzi, W. Liu, J. Weiland and M. Humayun, Computed SAR and thermal elevation in a 0.25-mm 2-D model of the human eye and head in response to an implanted retinal stimulator-part I: Models and methods, IEEE Transactions on Antennas and Propagation 51 (2003), 2274-2285.

[15] P. Bernardi, M. Cavagnaro, S. Pisa and E. Piuzzi, Specific absorption rate and temperature elevation in a subject exposed in the far-field of radio-frequency sources operating in the $10-900-\mathrm{MHz}$ range, IEEE Transactions on Biomedical Engineering 50 (2003), 295-304.

[16] A. Hirata, Temperature increase in human eyes due to near-field and far-field exposures at $900 \mathrm{MHz}, 1.5 \mathrm{GHz}$, and 1.9 GHz, IEEE Transactions on Electromagnetic Capability 47 (2005), 68-76.

[17] N. Makris, L. Angelone, S. Tulloch, S. Sorg, J. Kaiser, D. Kennedy and G. Bonmassar, MRI-based anatomical model of the human head for specific absorption rate mapping, Medical \& Biological Engineering \& Computing 46 (2008), $1239-1251$.

[18] S. Gabriel, R. Lau and C. Gabriel, The dielectric properties of biological tissues: III. Parametric models for the dielectric spectrum of tissues, Physics in Medicine and Biology 41 (1996), 2271-2293. 